[Mitteilungen aus dem II. Chemischen Universitätslaboratorium in Wien.]

\title{
Zur Kenntnis der Mellithsäure, Pyromellithsäure und ihrer Entstehung bei der Oxydation von Kohle: von Ernst Philippi.
}

(Eingelaufen am 24. Januar 1922.)

Den Anlaß zu den folgenden Arbeiten bot das Bedürfnis nach Pyromellithsäure, die ich zu meinen Arbeiten in der Dinaphthanthracenreihe sowie auch zu andern Synthesen benötigte. Kann man doch mit der Pyromellithsäure die meisten Reaktionen des Phthalsäureanhydrids, aber infolge der vier Carboxylgruppen doppelt ansführen. Zu der Zeit, als jene Arbeiten begonnen wurden (1911), war die Pyromellithsäure im Handel überhaupt nicht, im Laboratorium nur sehr umständlich und kostspielig beschaffbar.

Das Problem der Pyromellithsäuredarstellung ist aufs engste verwoben mit einer bequemen Zugänglichkeit der Mellithsäure; haben doch schon Erdmann ${ }^{1}$ ) und A. v. Baeyer ${ }^{2}$ ) erstere aus letzterer durch Abspaltung von 2 Mol Kohlendioxyd gewonnen:<smiles>O=C(O)C1CC(C(=O)O)C(C(=O)OCc2ccccc2)CC1C(=O)O</smiles>

Bei dieser Darstellungsweise ist es im allgemeinen geblieben.

ॠ) Journ. prakt. Chem. (1) 52, 432 (1851).

9) Ann. Suppl. 7, 1 (1870). 
Für die Mellithsäure wiederum hat in älteren Zeiten der in der Natur vorkommende Honigstein als Ausgangsmaterial gedient, eine Quelle, die aber infolge der immer seltener gewordenen Funde bald versiegte. Dafür beobachtete man aber das Auftreteu von Mellithsäure bei der Oxydation von Kohle mit Salpetersäure, Schwefelsäure und Chlorat, während sie bei der Oxydation mit Ozon, Sanerstoff oder Druckoxydation mit Luft nicht nachgewiesen werden konnte. Auch durch Oxydation der Seitenketten von tetra- und hexasubstituierten Benzolen mit den im Laboratorium gebräuchlichen Oxydationsmitteln wurde Mellithsäure und Pyromellithsäure wiederholt gewonnen, ohne dab dies zu einer einigermaßen brauchbaren Darstellungsmethode geführt hätte.

Unsere Untersuchungen sollten nun die bisher vorliegenden Literaturangaben nachprüfen, ergänzen und dadurch die Pyromellithsänre zu einer mit den gewöhnlichen Laboratoriumshilfsmitteln rasch und leicht zugänglichen Substanz machen. Da es an einer guten Methode zur Gewinnung von substituierten Pyromellithsäuren, die auf direktem Wege nicht erhältlich sind, fehlte, fiel auch dieses Ziel in den Bereich unserer Versuche.

Dementsprechend gliedert sich die folgende Abhandlung in das Studium der Oxydation von Kohle mit Salpetersäure, mit Schwefelsänre, die Ausarbeitung einer direkten Synthese der Pyromellithsäure aus technischem Xylol und endlich die Synthese von substituierten Pyromellithsäuren.

\section{I.}

Die Oxydation von Kohle mit Salpetersäure;

von Ernst Philippi und Gertrud Rie.

Zunächst galt es, nach einer möglichst einfachen und ergiebigen Darstellungsweise der Pyromellithsäure Umschan zu halten.

Sehr verlockend erschien das im Jahre 1911 an Read Holliday und O. Silberrad in Essex erteilte D.R.P. 
Nr. 214252, dessen Anspruch dahin lautet, daß feingepulverte Holzkohle mit Salpetersäure so lange gekocht wird, bis eine gelbe bis rotbraune Lösung entstanden ist, und dann die Salpetersäure bei einer $140^{\circ}$ nicht übersteigenden Temperatur abdestilliert wird. Der Trockenrückstand, im Gewichte etwas schwerer wie die angewandte Holzkohle, soll Mellithsäure von etwa 90 Proz. Reinheit sein. Die Nacharbeitung des Patentes durch den einen von uns bestätigte zwar die Angaben der beiden Engländer hinsichtlich Farbe und Menge des Rohproduktes; es gelang aber auf keine Weise daraus etwa durch Reinigung über verschiedene Salze Mellithsäure in nennenswerten Mengen zu erhalten. Ebensowenig war dies mit einem von den beiden Herren bereitwiligst zur Verfügung gestellten Originalpräparat möglich.

Da erschienen im Jahre 1914 die Arbeiten von Hans Meyer: „Zur Frage der Konstitution des Kohlenstoffmoleküls" ${ }^{\text {"1 }}$, und H. Meyer und K. Steiner: "Über die Mellithsäure" ${ }^{2}$ ), in denen ebenfalls die Oxydation von Holzkohle mit Salpetersäure beschrieben wurde. Das Verfahren dieser beiden Forscher unterscheidet sich von dem englischen im wesentlichen dadurch, daB die Salpetersäure nach Beendigung der Reaktion nicht bis zur Trockene des Rückstandes abdestilliert wird, sondern dab nach dem Verjagen nur eines Teiles der Salpetersäure aus der eingeengten Lösung eine erste reinere und dann noch eventuell eine zweite Krystallisation an Mellithsäure gewonnen wird. Zugabe von Vanadinsäure bei der Oxydation als Katalysator soll ebenfalls die Reaktion günstig beeinflussen. Was die Ausbeuten an dieser reineren, aber noch immer aschehältigen Mellithsäure betrifft, so gibt H. Meyer an, daß sie bei Anwendung vor Fichtenholzkohle etwa 40 Proz., Buchenholzkohle 15 Proz. der angewandten Kohle erhielten. Im übrigen seien die Aus-

1) Monatsh. 35, 163 (1914).

2) Monatsh. 35, 475 (1914). 
beuten recht schwankend, von der Provenienz der Kohle stark abhängig und konvergierten bei verschiedenen Laubholzkohlen, Braunkohle und Steinkohle gegen Null. Was die Reinigung dieser Rohmellithsäure anlangt, so blieben infolge der Unmöglichkeit einer Reinigung über den Ester noch drei Wege übrig: die Reinigung über Salze (das Ammonsalz ist in Wasser ziemlich schwer löslich, führt aber nur über das Baryt- oder Silbersalz za stickstoffreien Produkten), die Krystallisation aus Salpetersäure, oder endlich die Überführung der Rohmellithsäure in ihr Trianhydrid, welch letztere Methode gelegentlich von Meyer and Steiner empfohlen wird Aus den experimentellen Belegen geht aber deutlich hervor, daB die genannten Forscher die Hauptmenge ihres Rohproduktes über das Ammon- und Barytsalz reinigten. Von Interesse für die Beurteilung ihres Rohproduktes sind die von ihnen daraus erhaltenen Mengen an reinem Ammonsalz; sie berichten nämlich darüber folgendermaßen: $1 \mathrm{~kg}$ Fichtenholzkohle ergab $480 \mathrm{~g}$ Rohmellithsäure, aus der $240-320 \mathrm{~g}$ reines mellithsaures Ammonium erhalten wurden. Dies entspricht $120-160 \mathrm{~g}$ reiner krystallwasserfreier Mellithsäure, also 12-16 Proz. der angewandten Kohle. $1 \mathrm{~kg}$ Buchenholzkohle lieferte zwar auch $400 \mathrm{~g}$ Rohmellitbsäure, aus der sie aber nur $80 \mathrm{~g}$ reines Ammonsalz, entsprechend $40 \mathrm{~g}$ Mellithsäure isolieren konnten. In beiden Fällen konnten noch beträchtliche Mengen roher Barytsalze aus den Mutterlaugen gewonnen werden, von denen aber nicht bewiesen ist, dab es sich tatsächlich um mellithsaures Barium handelte. Immerhin konnte daraus bei der Destillation mit Schwefelsäure und Bisulfat Pýromellithsäure in guter Ausbente erhalten werden.

Soweit die Angaben von H. Meyer und Steiner. Wir entschlossen uns, ebenfalls diesen Weg zu beschreiten, und haben uns im Verlauf nnserer Untersuchung etwa $3 \mathrm{~kg}$ Rohmellithsäure nach diesem Verfahren hergestellt. Als Kohle verwendeten wir die offizinelle „Carbo ligni 
depuratus" ${ }^{\prime)}$ der österreichischen Heilmittelstelle, die laut Angaben der Pharmakopoea Austriaca aus Fichtenholzkohle besteht. Die einzige Abweichung gegeniiber den Angaben von Meyer und Steiner bestand darin, daB wir an Stelle von Salpetersäure vom spez. Gew. 1,51 mit solcher vom spez. Gew. 1,48-1,50 arbeiteten, da uns damals eine konzentriertere Säure in großen Mengen nicht zur Verfügung stand. Demgemäß war anch die Zeit bis zum Eintreten einer hellgelben Lösung etwas länger (nämlich statt 4 etwa 6-7 Tage) and wurde auch etwas mehr Säure hierzu benötigt. Unsere Ausbeuten an Rohmellithsäure (erste Krystallisation) betrugen im Mittel etwa 20-25 Proz. der angewandten Kohle, erreichten im günstigsten Falle 35 Proz. Aus den Mutterlaugen konnten wir noch beträchtliche Mengen, im Durchschnitt 10-15 Proz., Rohmellithsäure gewinnen. An den Rohprodukten, die hartnäckig Salpetersäure festhielten und in vacuo bei $160^{\circ}$ getrocknet wurden, fiel uns vor allem der hohe Aschengehalt auf, sowie der Umstand, daß die erste Krystallisation nicht hygroskopisch, das Produkt aus den Mutterlaugen aber hygroskopisch war.

Eine orientierende Analyse ergab für die erste Krystallisation (I und II), sowie das Produkt aus den Mutterlaugen (III und IV) folgende Werte:

I. 5,710 $\mathrm{mg}$ gaben $7,71 \mathrm{CO}_{2}, 1,355 \mathrm{H}_{2} \mathrm{O}$ und 1,03 Asche.

II. $31,93 \mathrm{mg}, 0,365 \mathrm{~cm}^{3}$ trocknes Stickgas bei $19^{\circ}$ und $743 \mathrm{~mm}$ Druck.

III. 5,272 $\mathrm{mg}$ gaben $8,085 \mathrm{CO}_{2}, 0,71 \mathrm{H}_{2} \mathrm{O}$ und 0,60 Asche.

IV. $11,14 \mathrm{mg} \# 0,415 \mathrm{~cm}^{3}$ trocknes Stickgas bei $19^{\circ}$ und $725 \mathrm{~mm}$ Druek.

\begin{tabular}{lccccc} 
& Ber. für & \multicolumn{4}{c}{ Gef. } \\
& $\mathrm{C}_{13} \mathrm{H}_{6} \mathrm{O}_{12}$ & I & II & III & IV \\
$\mathrm{C}$ & 42,11 & 36,86 & - & 41,84 & - \\
$\mathrm{H}$ & 1,77 & 2,65 & - & 1,50 & - \\
$\mathrm{N}$ & - & - & 1,31 & - & 2,42 \\
Asche & - & 18,04 & - & 11,51 & -
\end{tabular}

1) Der volle Name der österr. Pharmakopöe lautet: Carbo ligni pini depuratus in frustis. 
Die namentlich bei dem Produkt aus Mntterlaugen in die Augen fallende Tatsache, daß wir trotz eines Aschengehaltes von 11,5 Proz. und Stickstoffgehaltes von 2,4 Proz. innerhalb der üblichen Fehlergrenzen stimmende Werte für $\mathrm{C}$ und $\mathrm{H}$ erhielten, lehrt, dab unsere Produkte noch in beträchtlicher Menge Substanzen beigemengt enthalten, die wesentlich kohlenstoffreicher sind, als die Mellithsäure.

Wir gingen nun daran, die Reindarstellung der Mellithsäure aus dem Rohprodukt auf allen drei vorhin skizzierten Wegen zu versuchen, nämlich durch fraktionierte Fällung von Salzen, durch Umkrystallisieren aus Salpetersäure und über das Trianhydrid der Mellithsäure, das sich nach den Angaben von Meyer und Steiner auch aus mellithsaurem Natrium darstellen lassen sollte und bei dem daher keine Störung durch den hohen Aschengehalt zu erwarten war.

Die Reinigung über das Ammonsalz bzw. Barytsalz schlug vollkommen fehl. Wir erhielten zwar manchmal Krystalle des Ammonsalzes, aber stets nur in so geringer Menge, dab an ein Betreten dieses Weges für uns nicht zu denken war. Auch die wiederholte Krystallisation aus Salpetersäure führte uns nicht zum Ziele, nämlich zu einem Schmelzpunkts- und analysenreinen Präparat. Es ist uns unverständlich, daß Meyer und Steiner aus ihrem Rohprodukt durch Krystallisieren aus Salpetersäure direkt zu reinen Präparaten gelangten. Man erhält nämlich solche auch dann nicht, wenn man die Mellithsäure durch Oxydation von hexasubstituierten Benzolderivaten mittels Salpetersäure darstellt, wie wir im Falle der Oxydation von 1,3,5-Trimethyl-2,4-diäthyl6-acetylbenzol ${ }^{1}$ ) und Freund und Fleischer bei ihren schönen Indandionarbeiten ${ }^{2}$ ) beobachteten. Während die für Mellithsäure theoretisch geforderten Werte C:42,11 and $H: 1,77$ sind, erhielten Freund und Fleischer bei ihren Analysen $\mathrm{C}: 43,34,43,97$ und 43,42, $\mathrm{H}: 2,86,2,72$,

1) Monatsh. 42, 7 (1921).

) Diese Annalen 414, 22 ff. (1918). 
2,88, während wir bei unseren reinweißen, aber noch etwas zu tiefen Schmelzpunkt zeigenden Präparaten $\mathrm{C}: 43,53,43,07,43,03, \mathrm{H}: 2,34,2,12,2,02$ fanden. Erst die Reinigung über das Barytsalz lieferte uns ein schmelzpunktsreines Produkt mit den Werten $\mathrm{C}: 42,43, \mathrm{H}: 1,87$. Wenn also schon die reinweißen Oxydationsprodukte aus definierten Benzolhexaderivaten sich durch Umkrystallisation aus Salpetersäure nicht reinigen lassen, um wieviel schlechter sind dann die Aussichten bei den gelben, stark verunreinigten Produkten aus Kohle und Salpetersäure!

Wir betraten daher den dritten Weg, nämlich die Anhydrisierung der Mellithsäure. Diesbezüglich geben Meyer und Steiner an, dab man durch Erhitzen der Rohmellithsäure mit Acetylchlorid auf $160^{\circ} \mathrm{zum}$ Trianhydrid (I), durch Erhitzen mit Thionylchlorid auf die gleiche Temperatur aber zum Dianhydrid (II) gelangt, das vermöge der Parastellung der beiden freien Carboxyle nicht mehr befähigt ist, ein Trianhydrid zu geben.
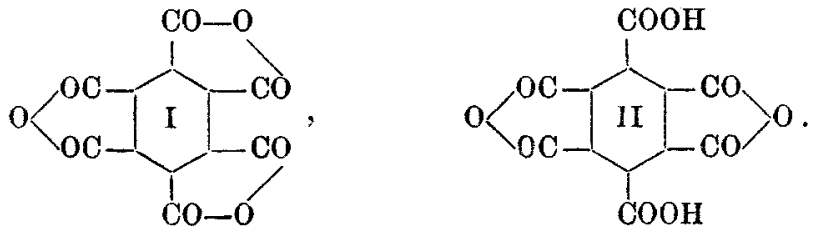

Die Reinigung des Trianhydrids sei schwierig, da es in allen gebräuchlichen Lösungsmitteln unlöslich ist und sich nur aus Acetylchlorid unter Druck oder aus sehr viel siedendem Benzoylchlorid umkrystallisieren läbt. Bei Drucken unter $4 \mathrm{~mm}$ soll es sublimierbar sein. Unser Rohprodukt, mit Acetylchlorid im Rohr auf $160^{\circ}$ erhitzt, ergab zwar glänzende Krystalle, die nach den Angaben mit trockenem Benzol gewaschen, sich aber als zum großen Teil anorganisch erwiesen. Jedenfalls gelang es nicht, daraus bei Drucken unter $4 \mathrm{~mm}$ ein Sublimat zu bekommen.

Wir wiederholten also unsere Versuche mit einwand- 
frei reiner Mellithsäure, die wir uns auf einem anderen Wege dargestellt hatten. ${ }^{1}$ ) Je $2 \mathrm{~g}$ reine, im Vakuum bei $120^{\circ}$ getrocknete Mellithsäure wurden mit $10 \mathrm{~cm}^{3}$ Acetylchlorid auf $150-160^{\circ}$ erhitzt. Beim Öffnen des Rohres entwich viel Chlorwasserstoff, glänzende helle Krystalle waren entstanden, die mit Benzol, in dem sie unlöslich sind, gewaschen und bei $110^{\circ}$ in vacuo getrocknet wurden. Die Analyse ergab folgendes:

I. $6,863 \mathrm{mg}$ gaben $11,82 \mathrm{CO}_{2}$ und $0,535 \mathrm{H}_{2} \mathrm{O}$.

II. $6,923 \mathrm{mg} \quad$ " $^{\prime} \quad 12,217 \quad \mathrm{CO}_{2}$ und $0,557 \quad \mathrm{H}_{2} \mathrm{O}$.

Ber. für

$\begin{array}{ccccc} & \mathrm{C}_{12} \mathrm{O}_{9} & \mathrm{C}_{12} \mathrm{H}_{2} \mathrm{O}_{10} & \text { I } & \text { II } \\ \mathrm{C} & 50,0 & 47,06 & 46,97 & 48,15 \\ \mathrm{H} & - & 0,65 & 0,87 & 0,90\end{array}$

Die gefundenen Werte deuten also auf ein Gemisch zwischen Di- und Trianhydrid hin, über deren Mengenverhältnis sich auf Grund der Elementaranalyse nicht viel aussagen läßt, da ja Zusätze von 10 Proz. der einen oder anderen Substanz noch innerhalb der üblichen Fehlergrenzen liegen. Wir suchten also ein rein chemisches Argument für die Auffassung unseres Produktes als Gemisch beizubringen, was wir dadurch bewerkstelligten, daß wir es nach Friedel-Crafts mit Aluminiumchlorid und einem Überschuß an Benzol in Reaktion brachten. Hierbei mübte aus dem Trianhydrid ein Gemisch von Tribenzoyltricarbonsänren, aus dem Dianhydrid ein solches von Dibenzoyltetracarbonsäuren entstehen. Tatsächlich gelang es uns, unser Reaktionsprodukt durch fraktionierte Krystallisation in zwei Fraktionen zu zerlegen, die zwar noch nicht einheitlich waren, aber doch bei der Elementaranalyse Werte ergaben, die sich bei der leichter löslichen Fraktion (I, II) den für Dibenzoyltetracarbonsäure verlangten, bei der schwerer löslichen Fraktion (III, IV) den für Tribenzoyltricarbonsäure geforderten, näherten.

1) Monatsh. 42, 7 (1921). 
I. 5,405 mg gaben $13,207 \mathrm{CO}_{2}$ und $1,855 \mathrm{H}_{2} \mathrm{O}$.

II. $6,410 \mathrm{mg} ", 14,88 \mathrm{CO}_{2}$ und $2,625 \mathrm{H}_{2} \mathrm{O}$.

III. $7,780 \mathrm{mg} \quad " \quad 18,84 \mathrm{CO}_{2}$ und $2,23 \mathrm{H}_{2} \mathrm{O}$.

IV. $5,800 \mathrm{mg} \quad, \quad 13,985 \quad \mathrm{CO}_{2}$ und $2,145 \mathrm{H}_{2} \mathrm{O}$.

Ber. für.

\begin{tabular}{lccrrrr} 
& $\mathrm{C}_{24} \mathrm{H}_{14} \mathrm{O}_{10}$ & $\mathrm{C}_{30} \mathrm{H}_{18} \mathrm{O}_{9}$ & I & II & III & \multicolumn{1}{c}{ IV } \\
$\mathrm{C}$ & 62,32 & 68,96 & 63,08 & 63,33 & 66,08 & 65,80 \\
$\mathrm{H}$ & 3,02 & 3,47 & 3,63 & 4,58 & 3,28 & 4,14
\end{tabular}

Wine Carboxylbestimmung durch Titration ergab ebenfalls Werte, die etwa in der Mitte zwischen den von Tri- und T'etracarbonsäuren verlangten lagen.

Wir glauben also damit den Beweis erbracht zu haben, daß beim Frhitzen von reiner Mellithsäure mit Acetylchlorid auf $150-160^{\circ}$ im Rohprodukt beide Anhydride nebeneinander entstehen.

Mit diesen Feststellungen war aber noch immer das Problem der Reinigung der aus Kohle und Salpetersäure erhaltenen Rohmellithsäure nicht gelöst. Andererseits konnten wir die Angabe von Meyer und Steiner, daß man aus der Rohmellithsäure bei der Destillation mit Schwefelsäure und Bisulfat Pyromellithsäure in bis zu 30 prozentigen Ausbeuten (also in fast ebenso großer Ausbente wie aus reiner Mellithsäure) erhält, vollauf bestätigen. Dies gilt auch für das eingangs erwähnte, uns von Holliday und Silberrad zur Verfügung gestellte Produkt. Daß aber das rohe Oxydationsprodukt keineswegs Mellithsäure von 90prozentiger Reinheit, wie von Holliday und Silberrad behanptet wird, darstellt, ist sowohl durch unsere Versuche wie auch durch die Resultate Hans Meyers, der ja auch im allergïnstigsten Falle nur 16 Proz. der angewandten Kohle an reiner Mellithsäure in Form ihres Ammonsalzes isolieren konnte, klar bewiesen. Bei der Buchenholzkohle sank diese Menge schon auf 4 Proz. herab und andere Kohlensorten gaben noch wesentlich schlechtere Ausbeuten. Unsere Produkte ergaben überhaupt auf keine Weise irgendwie nennenswerte Mengen an reiner Mellithsäure und erwiesen sich dennoch bei der Verarbeitung auf Pyromellithsäure als vollwertig. 
Nach unserer Ansicht ist die einzig mögliche Erklärung für diesen scheinbaren Widerspruch die, daß das aus Kohle und Salpetersäure direkt erhältliche Rohprodukt eben keine Mellithsäure darstellt, sondern ein Gemenge von verschiedenen Benzolpolycarbonsäuren, vornehmlich Mellithsäure, Benzolpentacarbonsäure und Pyromellithsäure, eventuell auch in geringerer Menge noch andere niedrigere Benzolcarbonsänren. Hierfür sprechen vor allem die bei unserem Rohprodukt gefundenen, in Anbetracht des Asche- und Stickstoffgehalts für Mellithsäure viel zu hoch liegenden Kohlenstoffwerte. Es ist auch ohne weiteres verstăndlich, daß ein Gemisch derartiger Säuren, die einander chemisch nahe stehen, durch fraktionierte Krystallisation, sei es der Salze oder der freien Säuren, nur schwer in einzelne reine Komponenten trennbar ist. Bei der Destillation mit Schwefelsäure und Bisulfat spalten aber die höheren Polycarbonsäuren, Mellithsäure und BenzoJpentacarbonsäure, Kohlendioxyd unter Pyromellithsăurebildung ab und man erhält auf diesem Wege ein einheitliches Reaktionsprodukt. Die Widersprüche zwischen den Angaben von Meyer und Steiner und unseren Resultaten hinsichtlich der Reinigungsmöglichkeit des Rohproduktes erklären sich aber zwanglos durch die Annahme, da $B$ das gegenseitige Mengenverhbiltnis der einzelnen Benzolpolycarbonsäuren variabel und durch geringfügige experimentelle Details bedingt ist, die wir gegenwärtig noch nicht meistern können. Meyer und Steiner hatten offenbar ein Rohprodukt in Händen, das an Mellithsäure reicher war wie unseres. Den Grund hierfür glauben wir aber weniger in der Art der angewandten Kohle suchen zu müssen, sondern wir denken, daß hierfür die Art der Ausführung der Oxydation, z. B. die Konzentration der Salpetersäure and damit die Temperatur verantwortlich sind, welche Ansicht durch die weiter unten beschriebene Oxydation von Kohle mit Schwefelsäure wesentlich gestïtzt wird. 
II.

\section{Die Oxydation von Kohle mit Schwefelsaure;}

von Ernst Philippi und Richard Thelen.

Die Einwirkung von Schwefelsäure auf Holzkohle ist schon lange bekannt und diente zur Darstellnng von schwefliger Säure; dabei wurde auch die Entstehung einer kleinen Menge einer "sublimierenden" organischen Substanz beobachtet. ${ }^{1}$ ) Während aber die früheren Beobachter den hierbei verbleibenden, wenig einladenden schwarzen und kohligen Rückstand nicht näher untersucht hatten, beschäftigte sich als erster A. Vernenil ${ }^{2}$ ) mit demselben. Es gelang ihm daraus in umständlicher und langwieriger $W$ eise darch fraktionierte Krystallisation and Fällung Benzolpolycarbonsäuren zu isolieren, von denen er Mellithsäure, Benzolpentacarbonsäure und Pyromellithsäure in beträchtlicheren Mengen, nämlich von je 1-2 Proz. der angewandten Kohle nachweisen konnte. Was die Mengenverhältnisse von Kohle und Schwefelsäure anlangt, so verwendete er geringere Mengen Schwefelsäure als der summarischen Oxydationsgleichung

$$
\mathrm{C}+2 \mathrm{H}_{2} \mathrm{SO}_{4}=\mathrm{CO}_{2}+2 \mathrm{SO}_{2}+2 \mathrm{H}_{2} \mathrm{O}
$$

entspricht, nämlich statt $1633 \mathrm{~g}$ nar $1400 \mathrm{~g}$ auf je $100 \mathrm{~g}$ Kohle. Denn die Bildung der Polycarbonsäuren erscheint inm abhängig von dem Mengenverhältnis der Schwefelsäure und von der Temperatur, bei der sich die Reaktion vollzieht. Die Hauptreaktion verläuft bis $280^{\circ}$, gegen Ende steigt die Temperatur auf $300^{\circ}$. Nach sechsstündiger Oxydationszeit wiegt der Kolbeninhalt 90-100 g. Hălt man diese Grenzen nicht ein, so hat dies ein Verbrennen des Produkts zur Folge und man erhält keine Benzolpolycarbonsäuren. Soweit die Angaben Verneuils.

Daß man beim Erhitzen von Kohle mit Schwefelsäure und darauffolgender Destillation mit Bisulfat kleiner Mengen

1) Bull. soc. chim. [2] 2, 413.

9) Compt. rend. 118, 195 (1894); 132, 1342 (1901). Bull. soc. chim. 25, 684 (1901). 
von Pyromellithsăure darstellen kann, hat auch schon Scholl beobachtet (Privatmitteilung), und H. Meyer erwähnt in seinen Arbeiten über die Pyromellithsäure (a. a. 0.), dab man sich auf diesem Wege die Pyromellithsaure rasch und bequem in Ausbeuten von 1-2 Proz. der angewandten Kohle darstellen kann.

In Verfolgung des bereits in der vorangehenden Arbeit skizzierten Gedankenganges, nämlich daB alle drei erwähnten Benzolpolycarbonsäuren bei der Destillation in Pyromellithsänre übergehen und man dabei den Vorteil hat, ein einheitliches Reaktionsprodukt $z \mathfrak{u}$ fassen, gingen wir daran, die Verneuilsche Reaktion näher zu studieren. Unser Ziel war, durch Variation der Vernenilschen Oxydationsbedingungen in bezug anf Menge und Konzentration der Schwefelsăure, Zeit und Temperatur, sowie Zugabe eines Katalysators auf rein empirischem Wege festzustellen, welche maximale Ausbeute an Pyromellithsäure sich eigentlich dabei erreichen liebe. Schon die ersten Versuchsreihen ergaben, daß als geeignetste Konzentration der Schwefelsäure eine Săure vom spez. Gew. 1,76-1,80 in Betracht kommt; stärkere Saure, etwa Olenm mit bedentendem Anhydridgehalt, eignet sich nicht. Das von Verneuil angewandte Mengenverhältnis von Kohle $1: 14$ erwies sich als noch immer zu hoch. Die besten Ausbeuten wurden bei dem Verhältnis 1:12 erhalten. Über den Einfluß

\begin{tabular}{|c|c|c|c|c|c|c|}
\hline \multirow{2}{*}{$\begin{array}{c}\text { Menge } \\
\text { der } \\
\text { Holzkohle }\end{array}$} & \multicolumn{2}{|c|}{ Schwefelsäure } & \multirow{2}{*}{$\begin{array}{l}\text { Kata- } \\
\text { lysator }\end{array}$} & \multirow{2}{*}{$\begin{array}{c}\text { Gesamt- } \\
\text { dauer der } \\
\text { Oxydation } \\
\text { in } \\
\text { Stunden }\end{array}$} & \multirow{2}{*}{$\begin{array}{l}\text { Erreichte } \\
\text { maximale } \\
\text { Tempe- } \\
\text { ratur }\end{array}$} & \multirow{2}{*}{$\begin{array}{l}\text { Ausbeute } \\
\text { an Pyro- } \\
\text { mellith- } \\
\text { säure } \\
\text { (Exsic- } \\
\text { cator } \\
\text { trocken) }\end{array}$} \\
\hline & Menge & spez. Gew. & & & & \\
\hline $100 \mathrm{~g}$ & $1200 \mathrm{~g}$ & 1,785 & - & $6^{8} / 4$ & $300^{\circ}$ & $4,9 \mathrm{~g}$ \\
\hline $100 \mathrm{~g}$ & $1200 \mathrm{~g}$ & 1,785 & - & 6 & $315^{\circ}$ & $6,6 \mathrm{~g}$ \\
\hline $100 \mathrm{~g}$ & $1200 \mathrm{~g}$ & 1,785 & $\mathbf{H g}$ & $61 / 9$ & $300^{\circ}$ & $6,3 \mathrm{~g}$ \\
\hline $100 \mathrm{~g}$ & $1200 \mathrm{~g}$ & 1,785 & $\mathbf{H g}$ & $51 / 2$ & $315^{\circ}$ & $8,2 \mathrm{~g}$ \\
\hline
\end{tabular}


der Temperatur und der Zugabe eines Tropfens Quecksilber als Katalysator orientiert am besten ein Blick auf vorstehende kleine Tabelle.

Damit war es gelungen, ein Verfahren zur Darstellung von Pyromellithsäure auszuarbeiten, das an Ausbeuten in bezug auf die angewandte Kohle ungefähr dasselbe leistete, wie die Oxydation mit Salpetersäure, jener gegenüber aber bedeutende Vorzïge in bezug auf Raschheit, Bequemlichkeit and Säureersparnis besitzt. Man braucht für je $100 \mathrm{~g}$ Kohle nur $1200 \mathrm{~g}$ rohe konz. Schwefelsäure gegenüber fast $2000 \mathrm{~g}$ rauchender Salpetersäure und gelangt statt in 4-7 Tagen schon nach einem 'Tage zum Ziel. Außerdem vermeidet man die recht unangenehme Entfernung hartnäckig anhaftender Salpetersäure durch Trocknen im Vakunm bei $160^{\circ}$, wobei es zur Entzündung und Verpuffen einzel er Chargen kommt, was na tirlich die Gesamtausbeute stark beeinträchtigt.

Nach dem nben beschriebenen Verfahren wurden im hiesigen Laboratorium einige hundert Gramm Pyromellithsäure von verschiedenen Experimentatoren hergestellt und. damit seine ullgemeine Brauchbarkeit erwiesen. Hierbei zeigte sich, daß die Ausbeute von etwa $8 \mathrm{~g}$ zwar öfters erreicht, aber nie wesentlich überschritten wurde und die Durchschnittsausbeuten bei einiger Übung $6-7 \mathrm{~g}$ betrugen.

\section{Darstellung von Pyromellithsäure.}

$1200 \mathrm{~g}$ rohe konz. Schwefelsäure (spez. Gew. 1,76 bis 1,80 ) werden mit $100 \mathrm{~g}$ fein gepulverter Holzkohle "Carbo ligni depuratus" auf $315^{\circ}$ in einem Rundkolben erhitzt. Ein mit einer Schutzhülse umgebenes Thermometer, welches in den Kolben taucht, zeigt die Temperatur während des ganzen Oxydationsvorganges an. Als Katalysator wird ein Tropfen metallischen Quecksilbers zugesetzt. Die Temperatur wird innerhalb 3 Stunden allmählich bis gegen $250^{\circ}$ gesteigert. Nach der dritten Stunde wird sie bis $315^{\circ}$ erhöht und zwar so, daß inner- 
halb einer Stunde $290-300^{\circ}$ erreicht werden. MaBgebend für die Geschwindigkeit der Temperatursteigerang ist der mitunter recht heftige Verlauf der stark schäumenden Reaktion, wobei ein Überschäumen verhindert werden muß. Auf der Temperatur $310-315^{\circ}$ wird so lange gehalten, bis das nunmehr ruhige, kleine Bläschen bildende Schäumen einem starken Spritzen, Blasenwerfen und Aufblähen der Reaktionsmasse Platz macht. Am Kolbenhals beginnen sich dann weiße, nadelformige Krystalle von Pyromellithsäureanhydrid anzusetzen. Die Reaktion im Kolben wird nun unterbrochen, der Kolbeninhalt mit möglichst wenig Wasser in eine Retorte gespült und 30-40 $\mathrm{g}$ Kaliumbisul fat hinzugefügt. Hierauf destilliert man die Pyromellithsä ure in eine mit Wasser gefüllte Vorlage aber. Die Ausbeute betrug im Durchschnitt 6-7 g Pyromellithsäure (im Exsiccator getrocknet).

\section{Anhang.}

Herr Heinrich Butterweck hat am hiesigen Institut die Verneuilsche Reaktion auch noch auf andere Substanzen übertragen. Er untersuchte natürliche Braunkohlen und Steinkohlen und stellte fest, dab sie bei der Oxydation mit Schwefelsaure annahernd die gleichen Ausbeuten an Pyromellithsäure lieferten wie Holzkohle. Auch "Schwefelsäurekohlen", die er durch Erhitzen von rein aliphatischen Korpern, wie Cellulose, Stärke, Paraffin und Glycerin mit Schwefelsăare darstellte, verhielten sich ebenso. Dies spricht dafür, dab für das endliche Auftreten von Benzolpolycarbonsäuren keine präformierten Ringsysteme nötig sind. Graphit, Tierkohle und KienruB erwiesen sich gegen Schwefelsäure derart widerstandsfähig, daß auch nach längerem Kochen mit Schwefelsäure schlieblich fast das ganze Ausgangsmaterial und die ganze Schwefelsäure wiedergewonnen werden konnten.

Eine Ergänzung zu diesem Kapitel bilden enḍlich. Amuten der Chenie 428. Bind. 
die Beobachtungen $H$. Schrötters ${ }^{1}$, daß auch aus Cholesterin, Cholalsäure und Terpenen beim Erhitzen mit Schwefelsäure Benzolpentacarbonsäure gebildet wird.

III.

Syuthese der Pyromellithsäure aus technischem Xylol; von Ernst Philippi, Reinhard Seka und Norbert Froeschl.

Während die vorangegangenen Abschnitte die Entstehung von Mellithsäure und Pyromellithsäure bei der Oxydation von Kohle durch Salpetersäure und Schwefelsäure zum Gegenstand haben, soll im folgenden über Versuche berichtet werden, die zu einer Synthese der Pyromellithsäure aus technischem Xylol führten. Versuche, die Pyromellithsäure durch Oxydation der Seitenketten von entsprechend konstituierten tetrasubstituierten Benzolen zu gewinnen, sind schon öfter, z. B. mit dem Durol $(1,2,4,5$-Tetramethylbenzol) gemacht worden. In neuerer Zeit haben Freund und Fleischer ${ }^{2}$ ) eine neue Darstellungsweise der Pyromellithsäure veröffentlicht, die vom p-Xylol ausging, das, acetyliert, zum 1,4-Dimethyl2-äthylbenzol reduziert, hierauf in 5́-Stellung neuerlich acetyliert und schließlich mit Salpetersäure im Rohr zu Pyromellithsäure oxydiert wurde. Diese Synthese hat aber den Nachteil der Schwerzugänglichkeit bzw. des hohen Preises von reinem p-Xylol. Ihre Übertragung auf das technische Xylol, das bekanntlich zum allergrößten Teile aus m-Xylol und nur zu etwa 5-10 Proz. p- und 2-6 Proz. 0-Xylol besteht, war von den genannten Autoren offenbar deshalb nicht versucht worden, weil sich in der Literatur eine von Victor Meyer ${ }^{3}$ ) stammende Angabe findet, daß bei der Acetylierung von m-Xylol nach Friedel-Crafts außer dem 1,3-Dimethyl4-acetylbenzol noch ein in 2-Stellung acetyliertes Diacetyl-

1) Monatsh. 29, 245, 395, 749 (1908).

2) Diese Annalen 414, 5 ff. (1918).

$\left.{ }^{3}\right)$ Ber. d. d. chem. Ges. 29, 2566 (1896). 
Zur Kenntnis der Mellithsäure usw.

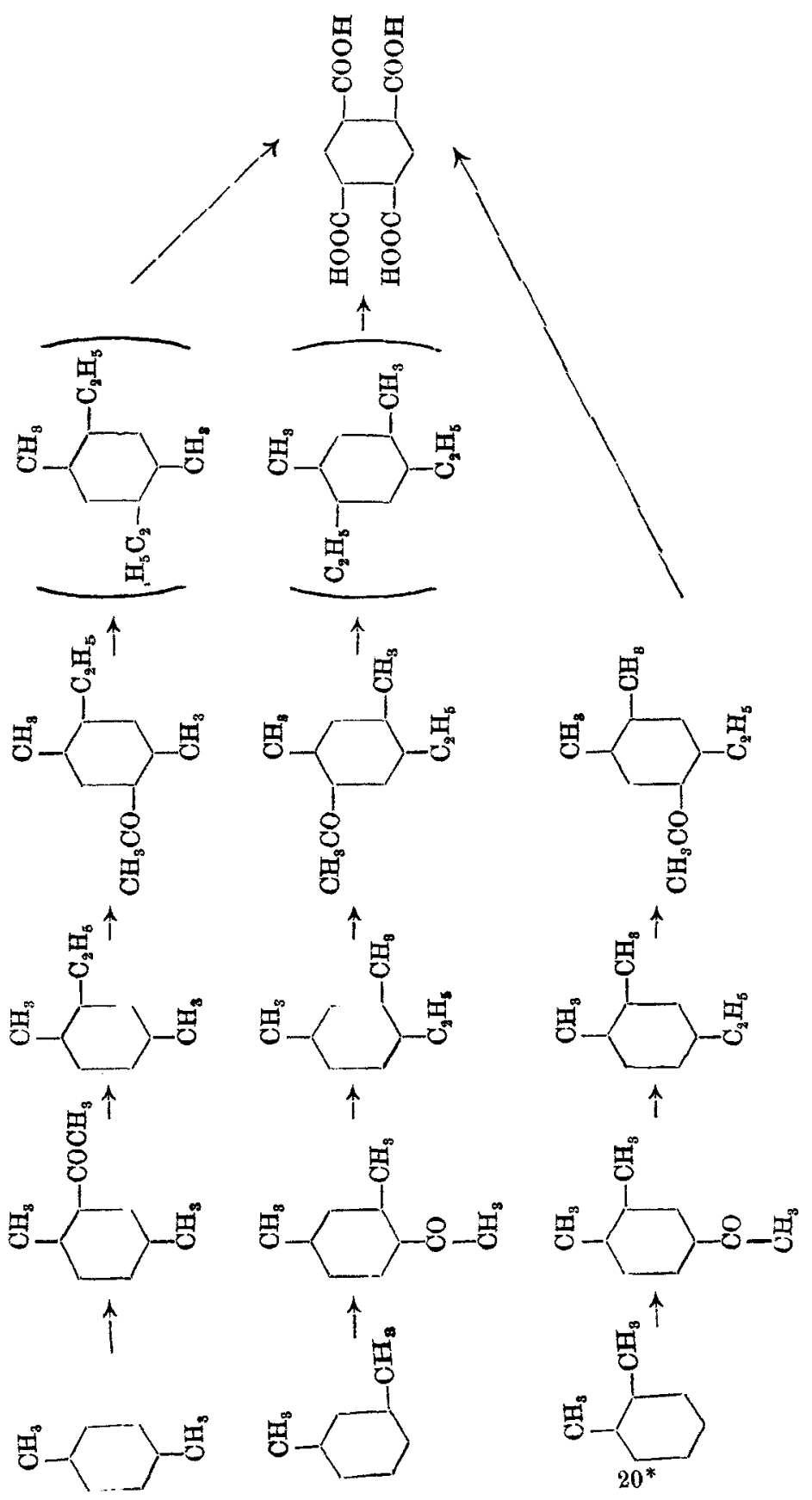


xylol als Nebenprodukt entstehen soll, während aus theoretischen Gründen vermöge des nach 0- oder p-Stellung orientierenden Finflusses der Substituenten bei den drei isomeren Xylolen vorstehender Reaktionsverlauf bei der stufenweisen Acetylierung za erwarten ist.

Es müBten also unter der Voraussetzung dieses Reaktionsverlaufes schlieBlich alle drei isomeren Dimethyläthylacetylbenzole als einheitliches Oxydationsprodukt Pyromellithsäure liefern.

Wir untersuchten zuerst den Reaktionsverlauf beim m-Xylol. Er entsprach tatsächlich unseren Erwartungen und es resultierte als einziges Oxydationsprodukt Pyromellithsäure, die außer durch den Schmelzpunkt und die Analyse, noch durch Überführung in ihr Anhydrid charakterisiert wurde. Hierbei stellten wir fest, da $B$ die Acetylierung beim $\mathrm{m}$-Xylol wesentlich leichter verläuft wie beim $p$-Xylol und $d a ß$ daher auch auf diesem Wege eine Anreicherung des $\mathrm{p}$-Xylols im technischen Xylol erreicht werden kann. Auch das technische Xylol ergab uns schlieblich als einziges Oxydationsprodukt Pyromellithsäure. Die Untersuchung des Acetylierungsverlaufes an reinem $0-\mathrm{Xyl}$ lol steht noch ans, und es bleibt daher vorläufig die Frage offen, ob das alleinige Auftreten der Pyromellithsäure im Falle des technischen Xylols durch einen Reaktionsverlauf im Sinne obiger Formelbilder bedingt ist, oder ob sich anders konstituierte, aus dem o-Xylol stammende Substitutionsprodukte infolge ihrer geringen Menge nicht bemerkbar machen.

\section{Versuche.}

1,3-Dimethyl-4-acetylbenzol (aus reinem m-Xylol von Kahlbaum).

$80 \mathrm{~g}$ Aluminiumchlorid werden mit Schwefelkohlenstoff uberschichtet und im Verlauf von $3 / 4$ Stunden ein Gemisch von $100 \mathrm{~g} \mathrm{~m}-X y l o l$ and $75 \mathrm{~g}$ Acetylchlorid zugegeben. Die Reaktion tritt entweder von selbst ein 
oder wird bei absolut trockenem Alaminiumchlorid (wie dies bei einem Präparat von den Wacker-Werken in Burghausen bisweilen vorkam) durch Zugabe eines Tropfens Wasser in Gang gebracht. Bisweilen war die Reaktion auch so heftig, daß gekühlt werden mußte. Hierauf wurden unter Umschütteln noch $20 \mathrm{~g}$ Aluminiumchlorid allmählich eingetragen. Nach längstens $11 / 2$ Stunden muß die Reaktion unterbrochen werden, da sonst Verharzung eintritt. Nun erfolgt die Aufarbeitung in normaler Weise, wobei man beachten muß, dab bei der Wasserdampfdestillation mit dem unveränderten $\mathrm{m} \cdot \mathrm{Xylol}$ anch nicht unbeträchtliche Mengen des Ketons $(6-8 \mathrm{~g})$ übergehen. Das Rohprodukt geht zwischen 225-240 über, bei nochmaliger Destillation siedet es bei $227-230^{\circ}$. Es erstarrt noch nicht bei $-20^{\circ}$. Die Konstitution wurde bereits durch Klaus ${ }^{1}$ ) durch die Oxydation zur $\mathrm{m}$-Xylylsäure als 1,3-Dimethyl-4-acetylbenzol bewiesen.

$5,90 \mathrm{mg}$ gaben $17,52 \mathrm{CO}_{9}$ und 4,42 $\mathrm{H}_{2} \mathrm{O}$.

$\begin{array}{ccr} & \text { Ber. für } \mathrm{C}_{10} \mathrm{H}_{12} \mathrm{O} & \text { Gef. } \\ \mathrm{C} & \mathbf{8 1 , 0 3} & 81,01 \\ \mathrm{H} & 8,17 & 8,38\end{array}$

Ausbente $64 \mathrm{~g}$ reines Keton ans je $100 \mathrm{~g}$ m-Xylol.

\section{1,3-Dimethyl-4-äthylbenzol.}

Die Reduktion des obigen Ketons erfolgte nach (lemmensen ${ }^{2}$ ), die Aufarbeitung in normaler Weise. Das Rohprodukt siedet zwischen $180-200^{\circ}$, das reine bei $184^{\circ}$ (unkorr.)

$5,47 \mathrm{mg}$ gaben $17,91 \mathrm{CO}_{2}$ und 5,39 $\mathrm{H}_{2} \mathrm{O}$.

$\begin{array}{ccc} & \text { Ber. für } \mathrm{C}_{10} \mathrm{H}_{14} & \text { Gef. } \\ \mathrm{C} & 89,52 & 89,33 \\ \mathrm{H} & 10,48 & 11,03\end{array}$

Ausbente $25 \mathrm{~g}$ reiner Kohlenwasserstoff aus $30 \mathrm{~g}$ Keton, d. i. 88 Proz. d. Th.

1) Ber. d. d. chem. Ges. 19, 230 (1886).

2) Ber. d. d. chem. Ges. 46, 1837 (1913). 


\section{1,3-Dimethyl-4-äthyl-6-acetylbenzol.}

Die Acetylierung erfolgte in derselben Weise wie beim m-Xylol; die Mengenverhältnisse waren diesmal $50 \mathrm{~g}$ Dimethyläthylbenzol, $30 \mathrm{~g}$ Acetylchlorid und im ganzen $50 \mathrm{~g}$ Aluminiumchlorid. Das Rohprodukt ging bei $20 \mathrm{~mm}$ Druck von $130-170^{\circ}$ über, bei wiederholter Destillation unter demselben Druck bei $140-150^{\circ}$. Bei starkem Abkühlen erstarrte es zu farblosen Nadeln, die nach dem Abpressen auf Ton bei $27^{\circ}$ schmolzen.

5,79 mg gaben 17,38 $\mathrm{CO}_{2}$ und 4,88 $\mathrm{H}_{2} \mathrm{O}$.

$\begin{array}{lcr} & \text { Ber. für } \mathrm{C}_{12} \mathrm{H}_{16} \mathrm{O} & \text { Gef. } \\ \mathrm{C} & \mathbf{8 1 , 7 6} & 81,91 \\ \mathrm{H} & \mathbf{9 , 1 5} & 9,31\end{array}$

Ausbeute $22 \mathrm{~g}$ reines Produkt, d. i. 64 Proz. d. Th.

\section{1,3-Dimethyl-4,6-diäthylbenzol.}

Die Darstellung dieser Verbindung erfolgte aus Gründen, die im Abschnitt IV der Arbeit angegeben werden. Die Reduktion erfolgte wieder nach Clemmensen. Das Rohprodukt zeigte bei $15 \mathrm{~mm}$ Druck den Siedep. $100-110^{\circ}$, das reine beim selben Druck $105^{\circ}$. Es erstarrte nicht bei $-20^{\circ}$.

$5,78 \mathrm{mg}$ gaben $18,77 \mathrm{CO}_{2}$ und 5,84 $\mathrm{H}_{2} \mathrm{O}$.

$\begin{array}{ccc} & \text { Ber. für } \mathrm{C}_{12} \mathrm{H}_{18} & \text { Gef. } \\ \mathrm{C} & \mathbf{8 8 , 8 2} & 88,59 \\ \mathrm{H} & \mathbf{1 1 , 1 8} & \mathbf{1 1 , 3 1}\end{array}$

Ausbeute 11,5 g Kohlenwasserstoff aus $20 \mathrm{~g}$ Keton, d. i. 62 Proz. d. Th.

Oxydation des 1,3-Dimethyl-4-äthyl-6-acetylbenzols zu Pyromellithsäure.

Je $2 \mathrm{~g}$ Keton wurden mit $7 \mathrm{~cm}^{3}$ Salpetersäure (spez. Gew. 1,32) und $2 \mathrm{~cm}^{3}$ Wasser 8 Stunden im Einschmelzrohr auf $130^{\circ}$ erhitzt. Nach dem Verjagen der Salpetersäure durch Eindampfen mit Wasser verblieb ein in Wasser fast unlöslicher Rückstand. Nach dem Umkry- 
stallisieren aus Eisessig zeigte er den Schmelzp. $266^{\circ}$ (unkorr.), der sich auch nach dem Sublimieren nicht anderte. Die Analyse dentet auf eine 1,3-Dimethyl4,6-benzoldicarbonsäure hin.

I. $6,065 \mathrm{mg}$ gaben $13,69 \mathrm{CO}_{2}$ und $3,04 \mathrm{H}_{2} \mathrm{O}$.

II. 6,32 mg (sublimiert) gaben $14,27 \mathrm{CO}_{2}$ und $2,60 \mathrm{H}_{2} \mathrm{O}$.

$\begin{array}{cccc} & \text { Ber. für } & & \text { Gef. } \\ & \mathrm{C}_{\mathbf{1 0}} \mathrm{H}_{10} \mathrm{O} & \text { I } & \text { II } \\ \mathrm{C} & 61,84 & 61,58 & 61,60 \\ \mathrm{H} & 5,19 & 5,60 & 4,61\end{array}$

Daraus geht hervor, daB die angewandten Reaktionsbedingungen noch nicht energisch genug waren, um die totale Oxydation zur Pyromellithsaure zu bewirken. Es wurden daher auf je $2 \mathrm{~g}$ Keton $10 \mathrm{~cm}^{3}$ Salpetersanare (spez. Gew. 1,32) unverdünnt angewandt und die Temperatur bis $200^{\circ}$ gesteigert. Nun resultierte ein in Wasser losliches Reaktionsprodukt, das sich als Pyromellithsäure erwies. Der Identitätsnachweis wurde außer durch den Schmelzpunkt und die Analyse auch noch durch die Überführung in das Pyromellithsäureanhydrid erbracht, um jede Verwechslung mit isomeren Benzoltetracarbonsäuren auszuschließen.

Analyse des aus Wasser amkrystallisierten Produktes vom Schmelzp. $264^{\circ}$ :

6,62 mg gaben 11,42 $\mathrm{CO}$, und 1,98 $\mathrm{H}, \mathrm{O}$.

$\begin{array}{ccc} & \text { Ber. für } \mathrm{C}_{10} \mathrm{H}_{6} \mathrm{O}_{8} & \text { Gef. } \\ \mathrm{C} & 47,25 & 47,06 \\ \mathrm{H} & 2,38 & 3,22\end{array}$

Analyse nach der Anhydrisierung mit Essigsăureanhydrid:

$5,925 \mathrm{mg}$ gaben $11,95 \mathrm{CO}_{9}$ und $0,73 \mathrm{H}_{2} \mathrm{O}$.

$\begin{array}{ccc} & \text { Ber. fur } \mathrm{C}_{20} \mathrm{H}_{\mathbf{2}} \mathrm{O}_{0} & \text { Gef. } \\ \text { C } & 55,05 & \mathbf{5 5 , 0 2} \\ \mathrm{H} & 0,92 & 1,37\end{array}$

Ausbeute im Durchschnitt $2,3 \mathrm{~g}$ Pyromellithsäure ans $2 \mathrm{~g}$ Keton, d. i. etwa 80 Proz. d. Th. 


\section{Technisches Xylol.}

Das käufliche Xylol wird fraktioniert und das von 137-142 ${ }^{\circ}$ übergehende Destillat verwendet. Fs empfiehlt sich die Acetylierung derartig $\mathrm{zu}$ bewerkstelligen, daß man an stelle des Schwefelkohlenstoffes als Verdünnungsmittel einen Überschuß des Xylols verwendet. Da das $\mathrm{m}$-Xylol von allen drei Isomeren am leichtesten acetyliert wird, so erreicht man dadurch, daß man im wesentlichen nur das Acetylprodukt des m-Xylols erhält. Die Trennung vom überschüssigen Xylol bei der Aufarbeitung erfolgt durch Destillation, wobei die Siedepunkte der einzelnen Acetylxylole folgendermaßen liegen: m-Acetylxylol $228^{\circ}$, p- bei 224-225 $5^{1}$ ) and o- nach den Angaben von Claus ${ }^{2}$ ) bei $243^{\circ}$.

Jie Reduktion und zweite Acetylierung verläuft vollkommen analog wie beim m-Xylol, ebenso die Oxydation mit Salpetersäure. Die Pyromellithsäure wurde in Form ihres Anhydrids vom Schmelzp. $283^{\circ}$ analysiert.

6,87 mg gaben $13,85 \mathrm{CO}_{2}$ und $0,88 \mathrm{H}_{2} \mathrm{O}$.

$$
\begin{array}{ccc} 
& \text { Ber. für } \mathrm{C}_{10} \mathrm{H}_{9} \mathrm{O}_{6} & \text { Gef. } \\
\mathrm{C} & 55,05 & 55,12 \\
\mathrm{H} & 0,92 & 1,47
\end{array}
$$

IV.

\section{Synthese ron substituierten Pyromellithsäuren;}

von Ernst Philippi, Reinhard Seka und Lilly Robinson.

Die Pyromellithsäure hat die Figenschaft, sich an den beiden noch am Benzolkern haftenden Wasserstoffen nicht direkt substituieren zu lassen. Wenigstens konnten wir in der Literatur keine diesbezüglichen Angaben finden. Der einzige, der substituierte Derivate der Pyromellithsäure beschrieben hat, war 1. U. Nef ${ }^{3}$ ), der über Nitro-, Amino- und Chinonderivate dieser Säure berichtet.

\footnotetext{
3) Ber. d. d. chem. Ges. 18, 1856 (1885).

2) Journ. prakt. Chem. 41, 409 (1890).

3) Diese Annalen 237, 19 ff. (1889).
} 
Sein Ausgangsmaterial war die Dinitropyromellithsäure, die er durch Nitrieren von Durol bzw. Durylsäure und darauffolgende Oxydation der Seitenketten erhielt; infolge der Schwerzugänglichkeit des Durols kommt aber dieser Synthese wohl keinerlei praktische Bedeutung zu. Die im Vorangehenden beschriebene stufenweise Acetylierung des Xylols, die schließlich zum Dimethyldiäthylbenzol führt, schien uns geeignet, diesen Übelstand zu beseitigen und auch die substituierten Pyromellithsäuren relativ leichter zugänglich zu machen.

Als Grundlage für unsere Versuche wählten wir das 1,4-Dimethyl-2-äthyl-5-acetylbenzol bzw. das 1,4-Dimethyl2,5-diäthylbenzol, die auf dem von Freund und Fleischer ${ }^{1}$ ) angegebenen Wege dargestellt wurden. Bei der Nitrierung dieser Substanzen stießen wir aber auf unerwartet große experimentelle Schwierigkeiten, die sich auch dadurch nicht beheben ließen, daß wir die größtenteils öligen, inhomogenen Nitrierungsprodukte gleich weiter zu den nitrierten Pyromellithsäuren oxydierten. Wohl konnten wir das symmetrische 1,4-Dinitro-2,5-dimethyl-3,6-diäthylbenzol, die Mononitro- und die Dinitropyromellithsäure isolieren, doch war die Reinigung der einzelnen Substanzen and die schließlichen Ausbeaten derart unerfreulich, daß wir diesen Weg aufgaben. Wir erklären diesen Mißerfolg damit, daß auch bei Anwendung möglichst starker Salpetersäure und einer $-10^{\circ}$ nicht übersteigenden Tempe. ratur stets neben der Nitrierung schon gleichzeitig teilweise Oxydation der Seitenketten erfolgt. Parallel mit dem Auftreten von Carboxylgruppen wird aber wieder die Nitrierung erschwert, die ja bei der Pyromellithsäure selbst überhaupt nicht zu erzwingen ist.

Wesentlich erfreulicher gestaltete sich aber die Bromierung von 1,4-Dimethyl-2,5-diäthylbenzol, die uns glatt zur Dibrompyromellithsäure führte, wobei wir anch

1) Diese Annalen 414, 5 (1918). 
den Reaktionsmechanismus der Oxydation im Sinne folgender Formelbilder verfolgen konnten:

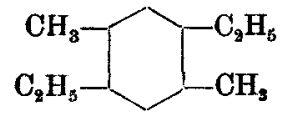<smiles>CCCCCCCC1C(Br)C(C)C(CC)C(Br)C1CC</smiles><smiles>CC1C(Br)C(C)C(Br)C(CC(=O)O)C1Br</smiles><smiles>CC1C(Br)C(C(=O)O)C(Br)C(C)C1OC(=O)O</smiles><smiles>O=C(O)C1C(Br)C(C(=O)O)C(C(=O)O)C(Br)C1C(=O)O</smiles>

Versuche.

Nitrierung von 1,4-Dimethyl-2,5-diäthylbenzol.

Bei den ersten Versuchen, bei denen Salpetersäure vom spez. Gew. 1,52 verwendet, teils nur unter Wasserkühlung, teils bei $-10^{\circ}$ gearbeitet wurde, resultierten bloß ölige Produkte, ans denen sich krystallisierte Substanzen nicht isolieren ließen. Folgende Arbeitsweise führte schlieblich allerdings in sehr schlechter Ausbeute zu symmetrisch konstituierten 1,4-I)initro-2,5-dimethyl3,6-diäthylbenzol.

$2 \mathrm{~g}$ Kohlenwasserstoff wurden unter Wasserkühlung zu 10 g Salpetersäure (D. 1,49) eintropfen gelassen und dann noch $8 \mathrm{~g}$ der gleichen Salpetersäure hinzugefügt. SchlieBlich wurde 3 Stunden am Wasserbad erhitzt. Auf Eis gegossen fällt das Nitrierungsprodukt in gelben Flocken aus, die abfiltriert, auf Ton abgepreßt und wiederholt aus verdünntem Alkohol umkrystallisiert wurden. 
Schließlich resultierte ein Produkt vom Schmelzp. $100^{\circ}$ (unscharf unter Verfärbung nach vorangehendem Erweichen).

4,310 $\mathrm{mg}$ gaben $0,441 \mathrm{~cm}^{3}$ trocknes Stickgas bei $20^{\circ}$ und $740 \mathrm{~mm}$ Druck.

N $\begin{array}{cc}\text { Ber. für } \mathrm{C}_{12} \mathrm{H}_{13} \mathrm{O}_{4} \mathrm{~N}_{2} & \text { Gef. } \\ 11,11 & 11,60\end{array}$

Nitrierung von 1,4-Dimethyl-2-äthyl-5-acetylbenzol.

Diese führte trotz mannigfacher Variation der Versuchsbedingungen nicht zu einheitlichen, definierten Körpern. Es gelang zwar, krystallisierte Produkte zu fassen und ihren Schmelzpunkt durch häufiges Umkrystallisieren aus verschiedenen Lösungsmitteln bis 159 bis $162^{\circ}$ hinaufzutreiben. Die Elementaranalysen ergaben aber stets Werte, die mit keiner rationellen Bruttoformel in Einklang zu bringen waren.

\section{Mononitro- und Dinitropyromellithsäure}

durch Nitrierung von 1,4-Dimethyl-2-äthyl-5-acetylbenzol and unmittelbare Oxydation des Rohproduktes mit Salpetersäure unter Druck.

Unsere zahlreichen Versuche in dieser Richtung wollen wir in Anbetracht ihres dürftigen Ergebnisses nur kurz wiedergeben. Sie führten zur Erkenntnis, daß gleichzeitig mit der Nitrierung stets auch schon Oxydation der Seitenketten erfolgte und daher keine einheitlichen Produkte entstehen.

Nitriert man mit der fünffachen Menge Salpetersäure (spez. Gew. 1,52) und geht bei der Oxydation im Rohr bis $160-170^{\circ}$, so läßt sich nach der Reinigung über das Kalksalz und wiederholtem Umkrystallisieren eine Substanz isolieren, deren Analyse auf Mononitropyromellithsäure hinweist:

I. 7,62 mg gaben $10,88 \mathrm{CO}_{8}$ und $1,30 \mathrm{H}_{2} \mathrm{O}$.

II. $5,58 \mathrm{mg}, 0,196 \mathrm{~cm}^{8}$ trocknes Stickgas bei $22^{\circ}$ und $749 \mathrm{~mm}$ Druck. 


$\begin{array}{ccc} & \text { Ber, für } \mathrm{C}_{10} \mathrm{H}_{5} \mathrm{O}_{10} \mathrm{~N} & \text { Get. } \\ \mathrm{C} & \mathbf{4 0 , 1 4} & 38,95 \\ \mathrm{H} & \mathbf{1 , 6 9} & \mathbf{1 , 9 1} \\ \mathrm{N} & 4,68 & 4,01\end{array}$

Die Schmelzpunkte lassen sich als Kriterium der Reinheit nicht verwenden, da, wie auch I. U.Nef (a.a. O.) angibt, nitrierte Pyromellithsäure sich bei $208^{\circ}$ unter Verfärbung allmählich zersetzt.

Zur Darstellung der Dinitropyromellithsäure ist ein großer Überschuß an Salpetersäure (die 10-12fache Menge der zu nitrierenden Substanz) erforderlich, und die Temperatur muß zur Oxydation bis $190^{\circ}$ gesteigert werden. Hierbei ist wiederholtes Herauslassen des enormen Druckes erforderlich. Unter Verlust eines großen Teiles der Substanz tritt tiefgreifende Zersetzung ein. Schließlich erhielten wir die Dinitropyromellithsäure als schwach gelbes Krystallpulver, das beim Erhitzen von $190-200^{\circ}$ unter Zersetzung schmolz, während I. U. Nef $208-225^{\circ}$ als Zersetzungspunkt angibt.

I. $8,035 \mathrm{mg}$ gaben $10,24 \mathrm{CO}_{3}$ und $1,00 \mathrm{H}_{2} \mathrm{O}$.

II. $5,845 \mathrm{mg} \quad, \quad 7,55 \mathrm{CO}_{2}$ und $1,05 \mathrm{H}_{2} \mathrm{O}$.

III. $2,785 \mathrm{mg}, \quad 0,206 \mathrm{~cm}^{3}$ trocknes Stickgas bei $26^{\circ}$ and $757 \mathrm{~mm}$ Druek.

Ber. fürr

$\begin{array}{cc} & \mathrm{C}_{10} \mathrm{H}_{4} \mathrm{O}_{18} \mathrm{~N}_{2} \\ \mathrm{C} & 34,88 \\ \mathrm{H} & 1,17 \\ \mathrm{~N} & 8,16\end{array}$

Gef,

$\begin{array}{ccc}\text { I } & \text { II } & \text { III } \\ 34,94 & 35,24 & - \\ 1,39 & 2,01 & - \\ - & - & 8,46\end{array}$

Endlich möchten wir noch erwähnen, daß wir im Verlaufe unserer Untersuchung auch eine Substanz erhielten, welche die von I. U. Nef für seine Dinitropyromellithsäure angegebene Zersetzungstemperatur zeigte. Weder diese Zersetzungstemperatur noch die elementare Zusammensetzung dieser Substanz änderte sich durch wiederholtes Umkrystallisieren oder Krhitzen mit Salpetersäure unter Druck auf $160-170^{\circ}$. Die Elementaranalysen der einzelnen Fraktionen lieferten stets an- 
nähernd konstante Werte (C 42,14 Proz., 41,71 Proz., 42,21 Proz.; H 2,03 Proz., 1,66 Proz., 3,38 Proz.; N 7,78 Proz., 7,04 Proz., 6,92 Proz., 6,83 Proz.), die wir aber mit keiner einigermaßen befriedigenden Strukturforme] in Eínklang zu bringen vermögen.

\section{1,4-Dibrom-2,5-dimethyl-3,6-diäthylbenzol.}

$100 \mathrm{~cm}^{3}$ Salpetersäure, D. 1,4, werden mit $100 \mathrm{~cm}^{3}$ Ligroin, Siedep. $80^{\circ}$, in dem $6 \mathrm{~g}$ Dimethyldiäthylbenzol gelöst sind, überschichtet, in einer Eis-Kochsalzkältemischang gekühlt und $60 \mathrm{~g}$ Schwefelbromür hinzutropfen gelassen. Anfangs mußte die Reaktion durch Eintauchen in lauwarmes Wasser eingeleitet, dann aber bald durch Kühlen auf $-15^{0}$ gemäbigt werden; nach $2 \frac{1}{2}$ stündiger Einwirkung wurden die beiden Schichten im Scheidetrichter getrennt, das Ligroin durch Schütteln mit Alkalilauge vom überschüssigen Brom befreit, mit Wasser gewaschen und getrocknet. Es resultierte eine gelbliche Lösung, die neben dem Bromkörper noch Schwefel und Bromkalium enthielt. Das Ligroin wurde verjagt und die hinterbleibende, gelbe Krystallmasse nach folgender Methode gereinigt. Zuerst wurde aus Eisessig umkrystallisiert; das nunmehr erhaltene Produkt, das noch immer Kaliumbromid und Schwefel enthielt, von diesen durch wiederholtes Aufnehmen in absoluten Äther befreit. Nach dem Eindunsten des Äthers erhielten wir schließlich das 1,4-Dibrom-2,5-dimethyl-3,6-diäthylbenzol in langen, weißen, schwach riechenden Nadeln. Es ist in Wasser sehr schwer, in Alkohol, Äther, Ligroin leicht löslich; aus Eisessig umkrystallisiert, zeigte es den Schmelzp. $81-82^{\circ}$ (unkorr.).

$\begin{array}{ccr}6,765 \mathrm{mg} \text { gaben } & 11,23 \mathrm{CO} \text { und } 3,02 \mathrm{H}_{2} \mathrm{O} . \\ 5,44 \mathrm{mg} " & \mathbf{6 , 4 1 5} \mathrm{AgBr} \text {. } \\ & \text { Ber. für } \mathrm{C}_{18} \mathrm{H}_{18} \mathrm{Br}_{2} & \text { Gef. } \\ \mathrm{C} & \mathbf{4 5 , 0 2} & \mathbf{4 5 , 2 9} \\ \mathrm{H} & 5,03 & 5,00 \\ \mathrm{Br} & 49,95 & 50,18\end{array}$


Oxydativer Abbau des 1,4-Dibrom-2,5-dimethyl3,6-diäthylbenzols.

$1 \mathrm{~g}$ Dibromdimethyldiäthylbenzol wird in einem Einschmelzrohr mit $4 \mathrm{~cm}^{3}$ Salpetersäure (spez. Gew. 1,35) 2 Stunden auf $140^{\circ}$ erhitzt; der starke Druck nach dem Abkühlen herausgelassen, hierauf 4 Stunden auf $160^{\circ}$ erhitzt. Beim Öffnen zeigt es sich, daß die Substanz in kleinen, zu Drusen vereinigten Nadeln ausgefallen ist, die in Wasser unlöslich sind. Es werden noch $2 \mathrm{~cm}^{3}$ Salpetersäure (spez. Gew. 1,42) hinzugefügt und neuerdings 2 Stunden auf $170^{\circ}$ erhitzt. Das Reaktionsprodukt wird durch dreimaliges Eindunsten am Wasserbad von der Salpetersäure befreit. Es hinterbleibt eine weiße Masse, deren Aufarbeitung folgendermaßen vor sich ging: es wird mit Wasser aufgekocht und vom Ungelösten abfiltriert.

Der ungelöste Rückstand, eine schwach gelblich gefärbte, in Nadeln krystallisierte Snbstanz, wird aus Äther umkrystallisiert; sie zeigt nach dem Trocknen im Vakuum bei $120^{\circ}$ keinen scharfen Schmelzpunkt, sondern beginnt sich von $180^{\circ}$ an unter Zersetzung dunkel zo färben. Wie aus den Analysen hervorgeht, ist es die 1,4-Dibrom-2,5-dimethylbenzol-3,6-diessigsäure.

I. $6,09 \mathrm{mg}$ gaben $8,41 \mathrm{CO}_{2}$ und $1,41 \mathrm{H}_{2} \mathrm{O}$.

II. $6,615 \mathrm{mg}, \quad 8,38 \mathrm{CO}_{\mathrm{g}}$ und $1,87 \mathrm{H}_{9} \mathrm{O}$.

III. $7,09 \mathrm{mg} " 6,89 \mathrm{AgBr}$.

\begin{tabular}{lcccc} 
& Ber. für & \multicolumn{3}{c}{ Gef. } \\
& $\mathrm{C}_{12} \mathrm{H}_{12} \mathrm{O}_{4} \mathrm{Br}$ r & I & U & III \\
$\mathrm{C}$ & 37,91 & 37,67 & 38,98 & - \\
$\mathrm{H}$ & 3,19 & 2,59 & 3,18 & - \\
$\mathrm{Br}$ & 42,07 & - & - & 41,95
\end{tabular}

Das Filtrat von der in Wasser unlöslichen 1,4-Dibrom-2,5-dimethylbenzol-3,6-diessigsăure zeigte nun folgende Eigenschaften: Beim Abkühlen fiel ein öl aus, das durch mehrmaliges Ausäthern extrahiert werden konnte. Nach dem Trocknen der ätherischen Lösung fielen beim Einengen des Äthers feine Krystallnadeln 
aus. Sie wurden aus Äther umkrystallisiert und zeigten den Schmelzp. $232^{\circ}$ (unkorr.). Es ist die 1,4-Dibrom2,5-dimethylbenzol-3,6-diketocarbonsäure.

$6,355 \mathrm{mg}$ gaben $8,27 \mathrm{CO}_{2}$ und $1,01 \mathrm{H}_{2} \mathrm{O}$.

$5,93 \mathrm{mg}, \quad 5,45 \mathrm{AgBr}$.

$\begin{array}{lcr} & \text { Ber. für } \mathrm{C}_{12} \mathrm{H}_{8} \mathrm{O}_{6} \mathrm{Br}_{2} & \text { Gef. } \\ \mathrm{C} & 35,30 & 35,50 \\ \mathrm{H} & 1,98 & 1,78 \\ \mathrm{Br} & 39,19 & 39,12\end{array}$

Die wäßrige Lösung wird nach dem Ausäthern eingedampft: es hinterbleibt ein weißer, hygroskopischer Rückstand, der bei $105^{\circ}$ im Vakuum getrocknet wurde. Zur Reinigung wurde er mit wenig Wasser aufgenommen, vom Ungelösten abfiltriert und im Vakuum eindunsten gelassen; hierbei schieden sich stark hygroskopische, leicht zerfließliche, rosettenartige Krystalle ab. Zur weiteren Reinigung wird in Äther gelöst, abfiltriert, der Äther eingedunstet und im Vakumm bei $110^{\circ}$ bis zur Gewichtskonstanz getrocknet. Schmelzpunkt: bei $170^{\circ}$ beginnt unter Dunkelfärbung eine.Veränderung vor sich zu gehen, zwischen 215 and $220^{\circ}$ wird die Substanz unter Verkohlung flüssig, um sich über $230^{\circ}$ unter lebhafter Gasentwicklung zu zersetzen.

Die Analyse erweist diesen Körper als Dibrompyromellithsäure:

$6,310 \mathrm{mg}$ gaben $6,80 \mathrm{CO}_{2}$ und $0,71 \mathrm{H}_{2} \mathrm{O}$.

$\begin{array}{crr}5,65 \mathrm{mg} & , \quad 5,105 \mathrm{AgBr} . & \\ & \text { Ber. für } \mathrm{C}_{10} \mathrm{H}_{4} \mathrm{O}_{8} \mathrm{Br}_{2} & \text { Gef. } \\ \text { C } & 29,14 & 29,40 \\ \mathrm{H} & 0,97 & 1,00 \\ \mathrm{Br} & 38,80 & 38,45\end{array}$

